\title{
Some recent progress in study of gravity and cosmology
}

\author{
LI Miao \\ Kavli Institute for Theoretical Physics, State Key Laboratory of Theoretical Physics, Institute of Theoretical Physics, Chinese Academy of Sciences, \\ Beijing 100190, China
}

Received September 20, 2012; accepted October 26, 2012

We briefly review some progress made in the areas of gravity and cosmology. Only a small portion of the work done in China is covered.

gravity, cosmology, dark energy

Citation: Li M. Some recent progress in study of gravity and cosmology. Chin Sci Bull, 2012, 57: 4668-4671, doi: 10.1007/s11434-012-5559-3

\section{Introduction}

The growth in economy in China in the last 20 years has been phenomenal. We have also witnessed the tremendous progress in developing fundamental research in China in the last decade. The area of gravity and cosmology is among the most rapidly developing directions in physics. We will briefly review some progress made in the last two years. It should be noted that only a small portion of the work done is mentioned.

The work in the area of gravity is mostly done in string theory, entropic gravity and physics of black holes; however we will only focus on the latter topics. The work in cosmology is even more diverse, and thus it is impossible to delve into all the various subareas of cosmology. We will only focus on dark energy.

In the next section, work in gravity theories is reviewed, and work concerning dark energy is reviewed in Section 3.

\section{Gravity theories}

There has been a number of papers written on entropic gravity in the last two years [1-9].

In $[1,2]$, the authors proposed to use the energy flow to replace energy in Verlinde's proposal to derive the Einstein equations. The obvious advantage of this proposal over the Verlinde's is that the holographic screen can be either closed or open, and one does not require it to be an equi-potential surface. Thus the derived Einstein equations are more general rather than valid only for some components. In this new proposal, one starts with the bulk energy flow through a disk $\Sigma:$

$$
\delta E=\int_{\Sigma} T_{\mu \nu} \xi^{\mu} N^{v} \mathrm{~d} A \mathrm{~d} t
$$

This is the expression in the $3+1$ dimensional bulk. The disk sweeps a solid cylinder in a time interal $\delta t$, and this cylinder has two kinds of boundaries: one consists of the initial disk and the final disk, and another is a cylinder swept by the boundary of the disk. From the viewpoint of holography, the energy flow can be expressed completely on the boundary. This expression can be divided into two parts. The first part is the change of energy on the disk due to change of the surface energy density, and the second part is the energy flow through the boundary of the disk.

By introducing the surface stress tensor $\tau_{i j}$, let $\xi^{i}$ be the Killing vector projected to the boundary, and let $u_{i}$ be the unit vector normal to the disk, $m_{i}$ be the unit vector normal to the cylinder. The energy flow on the surface can be expressed as

$$
\delta E=\left.\int u_{i} \tau^{i j} \xi^{j} \mathrm{~d} A\right|_{t} ^{t+\delta t}-\int m_{i} \tau^{i j} \xi^{j} \mathrm{~d} y \mathrm{dt}
$$




$$
=-\int_{\Sigma} D_{i}\left(\tau^{i j} \xi_{j}\right) \mathrm{d} A \mathrm{~d} t,
$$

where in the last equality we used the Stokes theorem. Now, both the bulk expression and surface expression of the energy flow have the same integral form. We need to equate the two integrands only. Introducing the assumption

$$
\tau^{i j}=c K^{i j}+q \gamma^{i j}
$$

where $K^{i j}$ is the extrinsic curvature of the surface and $\gamma_{i j}$ the induced metric, after some tedious calculations, we can derive

$$
c R_{\mu \nu}+f g_{\mu \nu}=T_{\mu \nu}, \quad q=-c K .
$$

Applying the Bianchi identity we can derive a formula for $f$ and setting $c=1 /(8 \pi G)$, and we obtain the Einstein equations with a cosmological constant. Thus the E.O.M. of the $f(R)$ gravity can be derived in a similar way, while Verlinde's original idea does not apply to a general $f(R)$.

In [3], another proposal is put forward. Let us use the first law of thermodynamics instead of the energy flow

$$
\delta S=\beta\left(\delta E-\omega^{a} \delta J_{a}+p \delta A\right) .
$$

Again, we work with a time-like screen. Now the screen is cylinder-like and encloses a solid cylinder. On the screen, the metric reads

$$
\gamma_{i j} \mathrm{~d} x^{i} \mathrm{~d} x^{j}=-N^{2} \mathrm{~d} t^{2}+\sigma_{a b}\left(\mathrm{~d} x^{a}+V^{a} \mathrm{~d} t\right)\left(\mathrm{d} x^{b}+V^{b} \mathrm{~d} t\right),
$$

where $x^{a}(a=1,2)$ and $\sigma_{a b}$ are coordinates and metric on $B$, respectively.

To calculate energy and angular momentum, we need surface energy density and current $j$; also the pressure is given by a tensor $s^{a b}$ :

$$
\begin{aligned}
& E=\int_{B} \mathrm{~d}^{2} x \sqrt{\sigma} \varepsilon, \\
& J_{a}=\int_{B} \mathrm{~d}^{2} x \sqrt{\sigma} j_{a}, \\
& \omega^{a}=\frac{V^{a}}{N}, \quad p^{a b}=s^{a b} .
\end{aligned}
$$

Thus, the first law on the surface reads

$$
\delta S=\beta \int_{B} \mathrm{~d}^{2} x\left[\delta(\sqrt{\sigma} \varepsilon)-\frac{V^{a}}{N} \delta\left(\sqrt{\sigma} j_{a}\right)+\frac{\sqrt{\sigma}}{2} s^{a b} \delta \sigma_{a b}\right] .
$$

We need a definition of temperature

$$
\beta=\left.i \int \mathrm{d} t N\right|_{B}
$$

with $t$ a pure imaginary number. Note that the above definition can apply to the cases without black hole and $\beta$ needs not to be a constant on the screen. We assume the local equilibrium conditions, so the temperature and other intensive quantities such as pressure and angular velocity can vary on the screen. For simplicity, we redefine (it) as $t$. Now $t$ is a real number and the first law eq. (2.10) becomes

$$
\begin{aligned}
\delta S= & \int \mathrm{d} t \int_{B} \mathrm{~d}^{2} x N\left[\delta(\sqrt{\sigma} \varepsilon)-\frac{V^{a}}{N} \delta\left(\sqrt{\sigma} j_{a}\right)\right. \\
& \left.+\frac{\sqrt{\sigma}}{2} s^{a b} \delta \sigma_{a b}\right] .
\end{aligned}
$$

The last ingredient is to introduce a surface stress so to have energy density and $j$ defined. If

$$
\tau_{i j}=c_{1} \bar{R}_{i j}+c_{2} \Theta_{i j}+f \gamma_{i j},
$$

then,

$$
\begin{aligned}
& \varepsilon=u_{i} u_{j} \tau^{i j}, \\
& j_{a}=-\sigma_{a i} u_{j} \tau^{i j}, \\
& s^{a b}=\sigma_{i}^{a} \sigma_{j}^{b} \tau^{i j} .
\end{aligned}
$$

Substituting the above into the first law, we find

$$
\begin{aligned}
\delta S= & \int_{{ }^{3} B} \mathrm{~d} t \mathrm{~d}^{2} x N\left[\delta(\sqrt{\sigma} \varepsilon)-\frac{V^{a}}{N} \delta\left(\sqrt{\sigma} j_{a}\right)+\frac{\sqrt{\sigma}}{2} s^{a b} \delta \sigma_{a b}\right] \\
= & \delta S_{0}+\frac{c_{2}}{2} \int_{M} \mathrm{~d}^{4} x \sqrt{-g}\left(R^{\mu \nu}-\frac{R}{2} g^{\mu \nu}+\Lambda g^{\mu \nu}\right) \delta g_{\mu \nu} \\
& -\frac{c_{2}}{2} \int_{\Sigma} \sqrt{h} \mathrm{~d}^{3} x\left(K h^{i j}-K^{i j}\right) \delta h_{i j} j_{t_{t^{\prime}}}^{t^{\prime \prime}} \\
& -\int_{{ }^{3} B} \mathrm{~d}^{3} x \sqrt{-\gamma} \delta\left(f+c_{2} \Theta+\frac{c_{1} \bar{R}}{2}\right) .
\end{aligned}
$$

Demanding $\delta S$ is a total differential, we find

$$
\begin{aligned}
& R_{\mu v}-\frac{R}{2} g_{\mu v}+\Lambda g_{\mu v}=0, \\
& \left.\delta h_{i j}\right|_{\Sigma_{t^{\prime}}}=\left.\delta h_{i j}\right|_{\Sigma_{t^{\prime \prime}}}=0, \\
& f=-c_{1} \frac{R}{2}-c_{2} \Theta+c_{3} .
\end{aligned}
$$

We reviewed only three papers published in the last two years. Numerous papers on entropic gravity have been written, and it is impossble to do justice to all of them. The work of [9] generalizes GKPW prescirption in the AdS/CFT correspondence to a more general spacetime, checking whether the thermodynamics work. This work may have a hidden link to the work we discussed above.

We will not review in detail the work done in physics of black holes. We refer to [10-13] for some papers in this exciting direction.

\section{Cosmology}

It appears that there are more people working in cosmology than in gravity in China, and thus it is not surprising that much more papers have been written in the past two years. Once again, it is impossible to even mention all the interesting work. We will focus on dark energy.

The longest review article on dark energy written by far is [14], and mostly recently this article is enlarged into a 
book [15]. The article and the book review comprehensively the field of dark energy, and cover both theory and numerical work.

Before we explain a new model of holographic dark energy with action, we would like to draw the reader's attention to a recent investigation on the nature of dark energy [16]. It is claimed in this paper that, while the cosmological constant appears consistent with current data, but a dynamical dark energy model which evolves from $w<-1$ at $z=0.25$ to $w>-1$ at higher red-shift is mildly favored. This result is obtained by applying a new non-parametric Bayesian method for reconstructing the evolution history of the equation-of-state $w$ of dark energy, to a collection of cosmological data. The latest supernova (SNLS 3-year or Union2.1), cosmic microwave background, red-shift space distortion and the baryonic acoustic oscillation measurements (including BOSS, Wiggle $\mathrm{Z}$ and $6 \mathrm{dF}$ ) are combined. Indeed, a similar result was obtained in [17-19]. If dark energy is indeed dynamical, this discovery will be as revolutionary as the discovery of dark energy itself. Of course, only time will tell whether the primitive evidence will turn out to be correct.

For other work on dark energy we refer the readers to [20-30].

Next, we briefly reveiw a most recent progress toward a holographic dark energy model (HDE) with actional principle. The first HDE model was proposed in [31], and this model by far is one of most successful dynamical dark energy models when data fitting is concerned. However, it has been frequently challenged that it possesses the causality problem and the circularity problem. The causality problem: it seems that the evolution of universe depends on the future information of universe, the future event horizon. Moreover, the equations of motion are non-local since the future event horizon is defined globally. The circular logic problem: the future event horizon exists only in an accelerating universe. How can one use an assumption based on the accelerating expansion to explain the accelerating expansion?

The resolution of the two problems in [32] is apparently simply by introducing an action for the holographic dark energy. Indeed one of the authors discussed this question with A. Zee some seven years ago and failed to find an action for the original HDE model. With a local action, apparently the causality problem can be avoided, since with a local action the derived equations of motion must be local as well. It is not surprising that when the causality problem is resolved, the circularity problem can be automatically resolved, since the event horizon will be determined by the initial conditions; therefore it must be finite.

The action proposed in [32] is given as

$$
\begin{aligned}
S= & \frac{1}{16 \pi} \int \mathrm{d} t\left[\sqrt{-g}\left(R-\frac{2 c}{a^{2}(t) L^{2}(t)}\right)\right. \\
& \left.-\lambda(t)\left(\dot{L}(t)+\frac{N(t)}{a(t)}\right)\right]+S_{M},
\end{aligned}
$$

where $R$ is the Ricci scalar, $a$ is the scale factor in the FRW metric, $N$ is the lapse function, and $a L$ is introduced to play the infrared cut-off in the HDE model. The E.O.M. derived from the above action are

$$
\begin{aligned}
\left(\frac{\dot{a}}{a}\right)^{2}+\frac{\kappa}{a^{2}} & =\frac{c}{3 a^{2} L^{2}}+\frac{\lambda}{6 a^{4}}+\frac{8 \pi}{3} \rho_{M}, \\
\frac{2 \ddot{a} a+\dot{a}^{2}+\kappa}{a^{2}} & =\frac{c}{3 a^{2} L^{2}}-\frac{\lambda}{6 a^{4}}-8 \pi p_{M},
\end{aligned}
$$

and

$$
\begin{aligned}
& \dot{L}=-\frac{1}{a}, \quad L=\int_{t}^{\infty} \frac{\mathrm{d} t^{\prime}}{a\left(t^{\prime}\right)}+L(\infty) \\
& \dot{\lambda}=-\frac{4 a c}{L^{3}}, \quad \lambda=-\int_{0}^{t} \mathrm{~d} t^{\prime} \frac{4 a\left(t^{\prime}\right) c}{L^{3}\left(t^{\prime}\right)}+\lambda(0),
\end{aligned}
$$

where we have set $N=1$. If we can prove $L(\infty)=0$ then we prove that the event horizon is a consequence of the solution of the e.o.m. We will see this shortly. The crucial acceleration equation is

$$
\frac{\ddot{a}}{a}=-\frac{\lambda}{6 a^{4}}-\frac{4 \pi}{3}\left(\rho_{M}+3 p_{M}\right),
$$

and it can be easily seen that eventually the first term always dominates the second in the far future. The dominating term is positive and thus the expansion of the universe eventually accelerates. Interesting, the intial value $\lambda(0)$ can be explained as dark radiation.

The asymptotic form of the solution reads

$$
a=c_{1} L^{\frac{1-\sqrt{1+\frac{4}{3} c}}{2}}+c_{2} L^{\frac{1+\sqrt{1+\frac{4}{3} c}}{2}} \sim L^{\frac{1-\sqrt{1+\frac{4}{3} c}}{2}} .
$$

Using $\dot{L}=-\frac{1}{a}$ and the asymptotic solution $a \sim L^{\frac{1-\sqrt{1+\frac{4}{3}} c}{2}}$, we can derive

$$
\begin{aligned}
& \frac{1-\sqrt{1+\frac{4}{3} c}}{3-\sqrt{1+\frac{4}{3} c}}, \quad c>6, \\
a & \sim e^{c_{3} t}, c_{3}>0, \quad c=6, \\
a & \sim\left(c_{4}-t\right)^{\frac{1-\sqrt{1+\frac{4}{3} c}}{3-\sqrt{1+\frac{4}{3}}}}, \quad c<6 .
\end{aligned}
$$

We see that in all cases the universe's expansion accelerates, the big rip occurs in the third case $c<6$. We also see that for $a \rightarrow \infty$ in the end; we must have $L \rightarrow 0$, thus proving $L(\infty)=0$. Therefore $a L$ is the event horizon and the causality problem is solved.

The exact solution with matter and radiation is presented in [32], and a numerical data fitting is already done and will be presented in a future paper.

\section{Conclusions}

We briefly reviewed some work in gravity and cosmology. We are witnessing tremendous research vigor in fundamental research force in these areas in China.

To end this review, we also refer the reader to recent papers in astrophysics [33-49]. Contained therein are applications to cosmology, for instance, work on the gamma-ray bursts. 
This work was supported by the National Natural Science Foundation of China (10535060/A050207, 10975172, 10821504).

$1 \mathrm{Gu}$ W, Li M, Miao R X. A new entropic force scenario and holographic thermodynamics. Sci China-Phys Mech Astron, 2011, 54: 1915-1924

2 Miao R X, Meng J, Li M. $f(R)$ gravity and Maxwell equations from the holographic principle. Sci China-Phys Mech Astron, 2012, 55: 375380

3 Li M, Miao R X, Meng J. Einstein equations from holographic thermodynamics and holographic entropy. arXiv: 1207.0661 [hep-th]

4 Ling Y, Wu J P. A note on entropic force and brane cosmology. J Cosmol Astropart Phys, 2010, 1008: 017

5 Kuang X, Ling Y, Zhang H. On thermal force from holographic action. arXiv: 1003.0195 [gr-qc]

6 Tian Y, Wu X N. Thermodynamics of black holes from equipartition of energy and holography. Phys Rev D, 2010, 81: 104013

7 Tian Y, Wu X N. Dynamics of gravity as thermodynamics on the spherical holographic screen. Phys Rev D, 2011, 83: 021501

8 Tian $\mathrm{Y}, \mathrm{Wu} \mathrm{X} \mathrm{N}$. Thermodynamics on the maximally symmetric holographic screen and entropy from conical singularities. J High Energy Phys, 2011, 1101: 150

9 Tian Y, Wu X N, Zhang H. Poor man's holography: How far can it go? arXiv: 1204.2029 [hep-th]

10 Zeng X X, Li L, Hu X Y. Entropy correction of charged black hole via fermions tunneling beyond semi-classical approximation. Sci ChinaPhys Mech Astron, 2010, 53: 116-121

11 Zhao R, Li H F, Zhang L C, et al. Radiation spectrum of a highdimensional rotating black hole. Sci China-Phys Mech Astron, 2010, 53: 504-507

12 Zhang J Y. Entropy correction of BTZ black holes in a tunneling framework. Sci China-Phys Mech Astron, 2010, 53: 1427-1433

13 Li H L, Deng Y F. Charged fermions tunneling radiation from the charged Godel black hole in minimal five-dimensional gauged supergravity. Sci China-Phys Mech Astron, 2010, 53: 1775-1779

14 Li M, Li X D, Wang S, et al. Dark energy. Commun Theor Phys, 2011 , 56: 525-604

15 Li M, Li X D, Wang S, et al. Dark Energy. Beijing: Peking University Press, 2012

16 Zhao G B, Crittenden R G, Pogosian L, et al. Examining the evidence for dynamical dark energy. arXiv: 1207.3804 [astro-ph.CO]

17 Li X D, Wang S, Huang Q G, et al. Dark energy and fate of the universe. Sci China-Phys Mech Astron, 2012, 55: 1330-1334

18 Zhang W S, Cheng C, Huang Q G, et al. Testing modified gravity models with recent cosmological observations. arXiv: 1202.0892 [astroph.CO]

19 Zhang Z, Li M, Li X D, et al. Generalized holographic dark energy and its observational constraints. Mod Phys Lett A, 2012, 27: 1250115

20 Huang T T, Wu P X, Yu H W. Observations favor the crossing of phantom divide lines. Sci China-Phys Mech Astron, 2010, 53: 562-566

21 Liu H, Li T P. New evidence for lack of CMB power on large scales. Sci China-Phys Mech Astron, 2010, 53: 567-570

22 Liu H, Li T P. Pseudo-dipole signal removal from WMAP data. Chin Sci Bull, 2011, 56: 29-33

23 Liu H, Li T P. Inconsistency between WMAP data and released map. Chin Sci Bull, 2010, 55: 907-909

24 Li M, Li X D, Zhang X. Comparison of dark energy models: A perspective from the latest observational data. Sci China-Phys Mech Astron, 2010, 53: 1631-1645

25 Guo H Y, Huang C G, Wu H T, et al. The principle of relativity, kinematics and algebraic relations. Sci China-Phys Mech Astron, 2010, 53: 591-597

26 Wu Y B, Yang W Q, Wang C, et al. Modified Chaplygin gas as an in- teracting holographic dark energy model. Sci China-Phys Mech Astron, 2010, 53: 598-606

27 Wang T S, Liang N. Constraints on Cardassian universe from Gamma ray bursts. Sci China-Phys Mech Astron, 2010, 53: 1720-1725

28 Li Y H, Ma J Z, Cui J L, et al. Interacting model of new agegraphic dark energy: Observational constraints and age problem. Sci ChinaPhys Mech Astron, 2011, 54: 1367-1377

29 Zhai Z X, Liu W B. Constraints of $f(R)$ gravity in Palatini approach with observational Hubble data. Sci China-Phys Mech Astron, 2011, 54: $1378-1383$

$30 \mathrm{Cao} \mathrm{S}$, Zhu Z H. The distance duality relation and the temperature profile of galaxy clusters. Sci China-Phys Mech Astron, 2011, 54: 22602264

31 Li M. A model of holographic dark energy. Phys Lett B, 2004, 603: 1-5

32 Li M, Miao R X. A new model of holographic dark energy with action principle. arXiv: 1210.0966 [hep-th]

33 Pan C J. Effects of radial velocity on line profiles and images from the standard thin disk. Sci China-Phys Mech Astron, 2010, 53: 155-162

$34 \mathrm{Xu} \mathrm{Y} \mathrm{D,} \mathrm{Ferrara} \mathrm{A,} \mathrm{Kitaura} \mathrm{F} \mathrm{S,} \mathrm{et} \mathrm{al.} \mathrm{Searching} \mathrm{for} \mathrm{the} \mathrm{earliest} \mathrm{galaxies}$ in the $21 \mathrm{~cm}$ forest. Sci China-Phys Mech Astron, 2010, 53: 1124-1129

35 Zhang Z Y, Gao Y, Wang J Z. CO observation of SNR IC 443. Sci China-Phys Mech Astron, 2010, 53: 1357-1369

36 Qin Y P, Gupta A C, Fan J H, et al. Duration distributions for different softness groups of gamma-ray bursts. Sci China-Phys Mech Astron, 2010, 53: 1375-1382

37 Zhang S N, Lu J F. Editorial. Sci China-Phys Mech Astron, 2010, 53(suppl. 1): 1-2

38 Liang E W. Gamma-ray bursts in the Swift-Fermi era: Confronting data with theory. Sci China-Phys Mech Astron, 2010, 53(suppl. 1): 14-23

39 Xin L P, Deng J S, Zheng W K, et al. Three years' systematic GRB follow-up at the Xinglong Observatory and the optical afterglow observations of GRB 080330. Sci China-Phys Mech Astron, 2010, 53(suppl. 1): $56-59$

40 Deng J G, Liang E W. Correlation between pulse-width and peak luminosity of single-pulse long gamma-ray bursts. Sci China-Phys Mech Astron, 2010, 53(suppl. 1): 69-72

41 Dong W, Liang E W, Lu R J. The flux-E-p relation within GRB060218 in comparison with typical GRB pulses. Sci China-Phys Mech Astron, 2010, 53(suppl. 1): 78-81

42 He H N, Wang X Y. The early high-energy afterglow emission from short GRBs. Sci China-Phys Mech Astron, 2010, 53(suppl. 1): 157160

43 Zhu L, Zhang S N. Diagnostics for the structure of AGNs' broad line regions with reverberation mapping data: Confirmation of the twocomponent broad line region model. Sci China-Phys Mech Astron, 2010, 53(suppl. 1): 196-201

44 Yuan J P, Wang N, Wu X J, et al. A mathematical method for the dedispersion of the pulsar profile. Sci China-Phys Mech Astron, 2010, 53(suppl. 1): 228-230

45 Xing Y, Yu W F. Decomposition of the X-ray waveform of soft gammaray repeaters during giant flares. Sci China-Phys Mech Astron, 2010, 53(suppl. 1): 249-251

46 Zhang $\mathrm{H}$ J, Zhao G, Zhang X, et al. The periodicity of 3C 273's radio light curve at $15 \mathrm{GHz}$ found by the wavelet method. Sci China-Phys Mech Astron, 2010, 53(suppl. 1): 252-255

47 Wang X, Huang Y F, Kong S W. Constraint on the counter-jet emission in gamma-ray burst after-glows from GRB 980703. Sci China-Phys Mech Astron, 2010, 53(suppl. 1): 259-261

48 Liu X W, Wu X F, Lu T. Magnetic energy injection in GRB 080913. Sci China-Phys Mech Astron, 2010, 53(suppl. 1): 262-264

49 Zheng W K, Deng J S. Evidence of a two-component jet in the afterglow of GRB 070419A. Sci China-Phys Mech Astron, 2010, 53(suppl. 1): $265-266$

Open Access This article is distributed under the terms of the Creative Commons Attribution License which permits any use, distribution, and reproduction in any medium, provided the original author(s) and source are credited. 